\title{
A High Level Architecture for Personalized Learning in Collaborative Networks
}

\author{
Hamideh Afsarmanesh and Jafar Tanha \\ Informatics Institute, University of Amsterdam, Science Park 107, \\ 1098 XG, Amsterdam, The Netherlands \\ h.afsarmanesh@uva.nl, j.tanha@uva.nl
}

\begin{abstract}
In Collaborative Network $(\mathrm{CN})$ environments, creation of collective understanding about both the aimed outcome and the procedure for achieving it by its members is the antecedent to any successful co-working and codevelopment. While a part of the common $\mathrm{CN}$ knowledge is pre-existing to its establishment, once the collaboration activities begin the emergent knowledge also needs to be commonly understood within this environment. Creating such commonality in understanding is however quite challenging. This paper suggests a bottom-up approach to reach collective understanding by all individuals involved in these networks, namely by the staff involved at all organizations which participate in the $\mathrm{CN}$. The proposed approach is founded on the idea of learning-together by the $\mathrm{CN}$ members to reach their collective understanding. In this approach, the domain/application experts in the $\mathrm{CN}$ act as the instructors and content providers, and assist with the modeling/remolding of the education domain for the $C N$ environment. Considering that the individuals involved in the $\mathrm{CN}$ are highly diverse and have different backgrounds, their learning requirements are also highly varied. Aiming to reach common understanding in CNs, this paper first addresses the main challenges in this area of learning; it then presents the related state-of-the-art and proposes a high level architecture for personalized learning of the members in collaborative networks.
\end{abstract}

Keywords: Personalized learning, Learning Ontology, Learning Style, Cognitive State, Swarm Intelligence.

\section{Introduction}

While beneficial to all CNs, creation of collective understanding is particularly important for the long term strategic CNs, e.g. the Virtual organizations Breeding Environments (VBEs), in which effective collaboration among its members are the base for innovation as well as improving the efficiency of the joint production of competitive goods and services. Nevertheless, considering the nature and representative characteristics of the VBEs, being a strategic alliance of organizations that covers wide variety of needed expertise within one sector or domain, its member organizations and their staff are highly diverse. For instance, each individual in the VBE, while is an expert in one specialty area (e.g. logistics, marketing, assembly, etc. in the manufacturing domain), may have little or no knowledge about other specialties in the VBE, or about the general knowledge of different collaboration models or existing support systems for it. 
Therefore every individual in the VBE has a different set of requirements for learning the pre-existing knowledge in this environment.

The base pre-existing knowledge common to all collaboration networks is currently addressed by: (1) the reference models defined for different kinds of CNs, e.g. applying the ARCON model (Afsarmanesh \& Camarinha-Matos, 2008), as well as (2) the base set of functionality supporting CN's management, e.g. the set of subsystems supporting the management of members' competency and trust, etc. in- the Virtual organizations Breeding Environments (VBEs) (Afsarmanesh, et. al., 2009). Finding the best learning materials as well as the near optimal learning path are the dreams of every learner, and these wishes are also shared by the learners in the $\mathrm{CN}$ environment. But unlike other learning environments, such as schools, which are typically characterized as individualistic and/or competitive learning environments (Johnson \& Johnson, 1999), learning within the $\mathrm{CN}$ environments needs to be a cooperative learning environment, due to the common goal shared by all learners in this environment, as needed to together achieve a bigger and more competitive mass in the market/society. Combining this specific required characteristic in the CNs with the wide diversity among the individuals in the CNs, creates yet another new challenge in this area. However, within a group representing the same competency/role/position in the $\mathrm{CN}$, e.g. design engineers, financial planners etc. the individuals have closer backgrounds, terminology, etc. than between such groups. Therefore, when it comes to the target area of learning inside the $\mathrm{CNs}$, further tailoring of the learning materials and the learning paths is also required according to specific learning characteristics of each group. This requires also addressing and modeling the personality, trait, or culture of the groups in regards to learning, which distinctly identifies one group against another. Considering both the vast amounts of potential materials on every subject, and the large number methods for learning, developing a personalized learning approach is promising.

In a face-to-face classroom, the instructor teaches a course simply using a textbook and a fix syllabus that covers the course in sequence. Instructors usually design a conventional learning path based on their personal understanding of what "good teaching" means as a result of their teaching experience. Learners then have to all follow a fixed list of contents. In such a case, since learners have minimal options among which to choose, their individual interests and preferences are generally ignored (Chen, liu, \& Chang, 2006). An alternative perspective was introduced for learning in the last decade, the aim of which was to provide personalized learning and find learning path customized to each learner, based on his/her preferences, requirements, learning style, and other determining characteristics.

Each learner has a specific Learning Style and a distinct Cognitive State and that LOs also have their own different Styles and Levels, etc. Ideally, when the learner has a request for learning, an automatic personalized learning system can generate the needed material and the guideline on what to study and in which order. This system careful considers his/her background knowledge and preferences etc. in order to identify the LOs whose specifications, better fit the learner's attributes, and provides the suitable learning path that best matches the learner style and considering the successful paths followed in the group to which the learner belongs. The problem which is explained above is primarily an optimization problem. The paper proposes the application of the Swarm intelligence method for solving this optimization problem, and 
finding suitable LOs and learning paths based on the specific collaboration among the learners and the group experiences.

The rest of this paper addresses the problem area of personalized learning in collaborative networks and its challenges and proposes a high level architecture to personalize the learning within collaborative networks.

\section{Related Research}

One line of research in this area is focused on personalized learning and finding suitable learning paths for learners. Some researches apply reasoning and semantic web techniques for personalized learning and recommendation of LOs, while others apply the optimization algorithms to find the best learning path and optimal solution. Other researches have also applied the multi agent system and intelligent tutoring to personalize learning. This paper however, due to the lack of space, addresses a few related researches, focused only on the first two main mentioned Approaches.

\subsection{Personalized Learning Based on Reasoning and Semantic Web}

PASER is proposed in (Kontopoulos et. al., 2008), and presents a system for automatically synthesizing curricula, applying the AI planning and semantic web methods. This system is based on the learner's profile, preferences, needs, and abilities to dynamically construct the learning path. However, this research does not adequately address personalized learning based on the learner's attributes and it also does not consider the features of LOs. In relation to our proposed approach, this research addresses the use of machine learning techniques for planning of the learning, but does not address any optimization algorithm. Also the proposed system does not indicate how this system is validated.

The research in (Gaeta et. al., 2009) describes methodologies and techniques for supporting a community of experts in modeling educational domains through the management of educational ontologies, namely through the e-learning ontologies and exploiting them in order to define and execute personalized learning experiences blended with learning activities. While the focus on the editor is strong, the issue of personalized learning is not sufficiently addressed by this research. Namely, it considers that identification of the learning path is simply done through a pre-existing graph, and does not address the detailed characteristics of the LOs, learners, or group experiences.

Research presented in (Chi, 2009) addresses a knowledge-intensive approach to create a general sequencing knowledge base for e-learning. This approach includes two components, including: (1) an ontology which is used to represent abstract views of content-sequencing and educational contents, and (2) a set of semantic rules that are used to represent relationships between individuals. However, this research does not adequately address the learners and LOs attribute. It mainly introduces a knowledge base system which based on its previously learnt knowledge suggests a learning path. Therefore its suggested learning path does not consider the set of features that we introduce in our proposed approach.

\subsection{Personalized Learning Based on Optimization Algorithm}

In (Chu et. al., 2009) the PC2PSO (personalized e-course composition based on Particle Swarm Optimization) is introduced for personalized learning, focused on four 
specific factors as its objective function. In their approach, Particle Swarm Optimization (PSO) method is used to find the near optimal solution, while almost entirely covering the above four objective functions. It is also discussed that when an e-course authoring tool is based on the proposed approach, the PC2PSO system facilitates instructors in selecting suitable e-learning materials from a mass repository. The issue of personalized e-course composition is however not sufficiently addressed by this research, because individual learner attributes is ignored. This research primarily aims to assist the instructor in finding suitable education material for suggesting to learners.

The research in (Wang \& Huang, 2008) proposes an extended approach based on the ant colony optimization, which is rooted in a recent Meta-heuristic algorithm for discovering the on-line learning patterns along an adaptive learning path. This research emphasizes the relationship between the learners' learning style and the leaning objects, in order to achieve an adaptive learning plan for each learner. However, the optimization algorithm introduced in this research does not consider the learner's cognitive state, and searching the LO is basically done within the static repository that sometimes is updated manually by instructors.

In (Lin et. al., 2009) an automatic course generation system is introduced for organizing the existing LOs in a repository. It applies a kind of swarm intelligence techniques namely, the Particle Swarm Optimization (PSO). The purpose of their research is to find solutions to the problem of how to assist instructors re-organize wellstructured teaching materials, utilizing existing repository of LOs. The suggested optimization algorithm in this research however does not consider the attributes of the learners and the LOs. This approach also only performs the search in a static repository and without consideration of resources accessible through the Internet.

In (Yang \& Wu, 2009) an attribute-based ant colony system is proposed for recommending the adaptation of learning objects for learners. It introduces an extended ant colony system based on the Kolb's learning style model (Kolb, 1974). The proposed system is used to construct a search mechanism for finding a suitable learning object. In this system fitting learner to the related learning object is achieved considering the learner's learning style and his/her domain knowledge, and also the LO's style and complexity level. In combinatorial optimization problems usually one important aspect is to find the adequate condition for stopping the iterative execution of the algorithm (due to time complexity issues). The approach suggested in this research however requires in some cases to comprehensively search all the nodes in the search space, which due to time complexity problems is not possible, without introducing any meta-heuristic technique to address this challenge. Also the search for LOs in this approach is done manually by the instructors. The proposed system needs to be validated in real environments.

\section{A High Level Architecture for Personalized Learning}

The comprehensive architecture proposed in this paper applies both of the above approaches for different purposes, and aims to identify and take into consideration the main determining features that influence personalized learning.

In the first step, we characterize the main features of the learners and the learning objects. The main features a learner include: (1) Learning style, (2) cognitive state, and (3) learning goals. Correspondingly, the important features identifying and 
describing a learning object include: (1) style, (2) level, and (3) educational objectives. These six features cover the most important aspects related to the problem area of personalized learning. Fig. 1 represents our proposed high level architecture, showing the main required modules for a personalized learning system and their primary interactions. The architecture represents a number of repositories storing different information about the learners, LOs, and the groups in the environment. One of these repositories deals with the LOs. A learning object is a resource, usually digital and web-based, that can be used and re-used to support the learning in the environment (IEEE-LOM). Each learning object has a Style (e.g. animation, text, diagram, and so on), a Complexity Level (e.g. very easy, easy, and etc.), and an educational objectives (addressing the subject it covers). For example in the VBE, the repository of LOs includes information related to the VBE management system functionalities (Afsarmanesh et. al. 2009). The individuals involved in a VBE need to learn about these general aspects, but at different levels of details depending on their role and tasks in the VBE. As a result, careful construction of the LOs' repository in collaborative networks is important and necessary for its effective operation, as explained in the Introduction section.

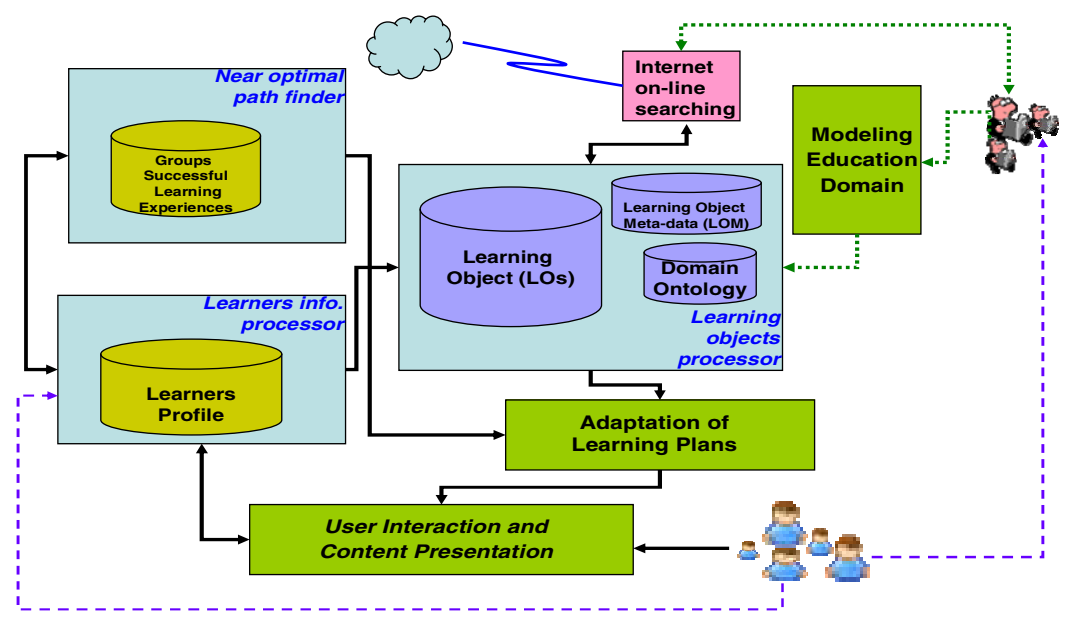

Fig. 1. A high level architecture for personalized learning in collaborative networks

Learning Object Metadata (LOM) is a data model, usually encoded in XML and RDF (IEEE, 2002 \& IMS, 2004), and is used to describe a learning object and similar digital resources that support learning. The purpose of LOM is to support the reusability of learning objects, to aid discoverability, and to facilitate their interoperability, usually in the context of online learning. In our approach the LOs are indexed by LOM in order to let the system know about their attributes and how they can be used. Therefore, the LOM repository is another important component in this architecture. Each LOM describes: (1) a list of explained concepts, (2) property of each concepts and its special value, and (3) a list of learning objects in the repository. 
A third repository in the architecture is an ontology, which represents all Domain Concepts (DC) and their inter-relationships. As such, a DC is any concept belonging to the described educational domain and is possibly explained by several LOs.

The repository of the learners profile consists of the user's information, such as his/her cognitive state, learning goals, and learning style (Kolb, 1974 and Felder \& Silverman, 1988). The last repository contains information which is gathered for each group in the $\mathrm{CN}$, during the operation of its learning system. Namely, this repository collects and records the information about the paths followed in any successful learning experience by any member of each group. The content of this repository is very important for further adaptation of the learning plan for the individuals in each group.

The learner model in this system is composed of learner's Cognitive State, Learning style, and learning goals. Cognitive state represents the current knowledge of the learner on the specific topic which he/she tries to learn. Each learner also has a preferred method for learning, which is called the learning style (Felder 1988). Meanwhile, each education has an objective; similarly each learner also has his/her learning goals.

The Modeling of Educational domain is a module in the architecture to assist the instructors and content providers to set up the learning domain for the CNs. For instance, through this domain, the instructors can create relationships between the ontology and LOMs. Through this module also a metadata is associated to one or more learning objects that exist in the LOs repository. Instructors and content providers in a specific domain construct the domain concepts in the ontology. Then they create relation between LOs and concepts.

The Near Optimal path finder tries to find for each subject learned in each group, the successful learning path followed by the most number of learners who passed the test on that subject. So based on the past experiences, the system learns to suggest better paths for future requests. In fact the feedback reports of the learners in each group on each subject, which is included in their profile, will be processed in the background by this path finder to provide the successful path information for the repository.

The Adaptation of learning Plans module try to find the best learning path for the learner from a group in the $\mathrm{CN}$ who tries to learn a subject. Imagine the learner submits a list of Target subjects to study. Given the Target $\mathrm{C}$ as input, the planning model builds a list of LOs which satisfy all the Target C's DCs, taking into account the learner's cognitive state, learning style, and learning goals. In this model also all restrictions and attributes of the LOs are considered. The module looks at all these attributes as aspects within an optimization problem. In our approach applying the Swarm intelligence techniques such as the Particle Swarm Intelligence (Kennedy, 1995) or Ant colony system (Dorigo, M., 1992) are foreseen to serve as the best methods for finding the near optimal solutions.

The User Interaction and Content Presentation module is the interface through which both the user specifies his/her request, and the system presents to user the learning path and the list of LOs, as a response to his/her request. In fact presentation of a response to the user consists of a relevant list of LOs delivered to the learner, while addressing all her/his learning requirements.

The leaner interacts with the system for personalized learning. According to the Fig. 1, users submit their request, and then based on their current knowledge on the subject and/or domain that they need to learn, the system identifies and presents a list 
of LOs together with a learning path. In time, the profile of each learner is updated with the feedback from the learners test results. Also learners participate in evaluating the subject they learned, through commenting on the LOs, as well as other aspect related to the performance of the system. This feedback will be then processed by the content providers and instructors.

\section{Further Challenges in Personalized Learning}

As was indicated in the description of the high level architecture of Figure 1, there are a number of challenges that need to be addressed for personalized learning in CNs. Below these challenges are further addressed.

An important part of the personalized learning is how to deal with the learners' model. In this model recognizing the current knowledge of the learner in relation to the domain/subject which he/she tries to learn is challenging, because the identification and evaluation of the level of human knowledge is complex. Each learner has a different learning goals and different preference for the learning style. But, similar to above, identification and formulation of both the preferred learning style and the learning goals of the learners are challenging. .

Another important feature of the personalized learning is how to identify the list of learning objects that best match the learners' requirements. On one hand, there are learners' aims and profile, and on the other hand there exist a large number of LOs addressing the subject in question. Developing the suitable technique to fit these two aspects and finding the near optimal solution is challenging. Similarly identification of the near optimal path for the learner requires its input from the results of mining all past successful learning experiences in different groups.

Another challenge in this architecture is how to deal with expanding the learning objects available in the system with the LOs that can be found on the web, namely how to search for LOs on the Internet. For instance if a subject requested by a learner is not sufficiently addressed in the existing repositories, then identifying the set of criteria to be used for searching/mining new suitable LOs on the Internet, and primarily based on the learner's profile, is challenging.

The architecture also represent collecting feedbacks from the learners. Voluntary input from learners about learning materials (indicated by the dashed arrow on the right hand side of Figure 1) helps the instructors to improve the Ontology, LOM, and LOs. With the results of learners' on the subject (indicated by the dashed arrow on the left hand side of Figure 1), the cognitive states of the learners in their profiles will be updated, and near optimal learning paths for future learners will be adjusted.

\section{Conclusion}

E-leaning, on-line, and web-based tutoring systems supporting the learning process is a promising approach in creation of collective understanding on pre-existing knowledge in CNs. In collaborative networks, such as in VBEs, the involved individuals are not typical learners in academic environments, and therefore it is even more important to make the learning time/cost effective. The personalized learning approach suggested in this paper aims to carefully apply both the learner's and the environment's 
characteristics into the customization of the most suitable reduced list of LOs, as well as the near optimal learning path for each learner individually. The paper addresses this problem area, its key conceptual challenges, and proposes a high level architecture to personalize the learning within CNs. This bottom-up approach represents the first step required for building the $\mathrm{CN}$ 's common terminology and later on the ontology of the environment, as subjects of forthcoming papers.

\section{References}

Afsarmanesh, H., Camarinha-Matos, L.M.: The ARCON modeling framework. In: Collaborative networks: reference modeling, pp. 67-82. Springer, New York (2008) ISBN: 978-0387-79425-9

Afsarmanesh, H., Camarinha-Matos, L.M., Msanjila, S.S.: On Management of 2nd Generation Virtual Organizations Breeding Environments. Journal of Annual Reviews in Control 33(2), 209-219 (2009)

Chen, C.-M., Liu, C.-Y., Chang, M.-H.: Personalized curriculum sequencing utilizing modified item response theory for web-based instruction. Expert Systems with Applications 30(2), 378-396 (2006)

Chi, Y.-L.: Ontology-based curriculum content sequencing system with semantic rules. Expert Systems with Applications 36, 7838-7847 (2009)

Chu, C.-P., Chang, Y.-C., Tsai, C.-C.: PC2PSO: personalized e-course composition based on Particle Swarm Optimization. Appl. Intell. (2009), doi:10.1007/s10489-009-0186-7

Dorigo, M.: Optimization, learning and natural algorithms, $\mathrm{PhD}$ thesis, Politecnico di Milano, Italy (1992)

Felder, R.M., Silverman, L.K.: Learning and teaching styles in engineering education. Engineering Education 78(7), 674-681 (1988)

Gaeta, M., Orciuoli, F., Ritrovato, P.: Advanced ontology management system for personalised e-Learning. Knowledge-Based Systems 22, 292-301 (2009)

IEEE, Standard for learning object metadata: http: / / 1 tsc . ieee. org/wg12

IMS, Learning Resource Meta-data Information Model Version 1.2.2, IMS Global Learning Consortium (2004), http: / / www. imsglobal org/

Johnson, D.W., Johnson, R.T.: Learning Together and Alone: Coopeartive, Competitive, and Individualistic Learning. Allyn \& Bacon, Boston (1999)

Kennedy, J., Eberhart, R.: Particle swarm optimization. In: Proceedings of IEEE international conference on neural networks, vol. 4, pp. 1942-1948 (1995)

Kolb, D.A.: Learning style inventory technical. McBer \& Co., Boston (1974)

Kontopoulos, E., Vrakas, D., Kokkoras, F., Bassiliades, N., Vlahavas, I.: An ontology-based planning system for e-course generation. Expert Systems with Applications 35, 398-406 (2008)

Lin, Y.-T., Cheng, S.-C., Yang, J.-T., Huang, Y.-M.: An Automatic Course Generation System for Organizing Existent Learning Objects Using Particle Swarm Optimization. In: Chang, M., Kuo, R., Kinshuk, Chen, G.-D., Hirose, M. (eds.) Learning by Playing. Game-based Education System Design and Development. LNCS, vol. 5670, pp. 565-570. Springer, Heidelberg (2009)

Wang, T.-I., Wang, K.-T., Huang, Y.-M.: Using a style-based ant colony system for adaptive learning. Expert Systems with Applications 34, 2449-2464 (2008)

Yang, Y.-J., Wu, C.: An attribute-based ant colony system for adaptive learning object recommendation. Expert Systems with Applications 36, 3034-3047 (2009) 